\title{
Special Issue on Radio Access Network Architectures and Resource Management for 5 G
}

\author{
J. Pérez-Romero, X. Lagrange, J. Nasreddine, J. Marquez-Barja
}

As a next step in the evolution of mobile communication systems, research carried out by industry and academia, is currently focused on the development of the new generation of mobile and wireless systems, known as 5th Generation (5G) with a time horizon beyond 2020. 5G will provide solutions to the ever-increasing demand for mobile services: the proliferation of bandwidth-intensive applications including high definition video, 3D and virtual reality, the expected increase of Machine To Machine (M2M) communications, the need for extremely reliable communications for services such as e-health, etc. To cope with the abovementioned demands, the requirements of future $5 G$ systems have been already identified at different fora. Some of these requirements include 1000 times higher wireless area capacity, 10 to 100 times higher number of connected devices, 10 to 100 times higher user data rate, 10 times longer battery life for low power Massive Machine Communication devices, and 5 times reduced End-to-End (E2E) latency.

The fulfilment of these challenging requirements together with the broad range of identified use cases and scenarios in $5 \mathrm{G}$ will demand more than a simple evolution of the radio access technology or the network architecture like in previous generations such as 2G/3G/4G. Instead, more radical changes can be expected from the very beginning, even in the main concepts of cellular networks. These changes are needed to provide the necessary degree of flexibility that allows combining heterogeneous components of $5 \mathrm{G}$ under a common holistic framework. In this respect, the introduction of Network Function Virtualisation (NFV) and Software Defined Network (SDN) capabilities are also expected to play an important role in the development of the future $5 \mathrm{G}$ networks.

Under the aforementioned general framework, and considering that nowadays the research towards 5G solutions is still in its initial stage, this special issue focuses on the radio access part. More specifically, it provides insights on the new architectures and resource management techniques that will allow achieving the requirements expected for future $5 \mathrm{G}$ networks. For that purpose, a total of five papers have been selected addressing different topics that are relevant from the perspective of the $5 \mathrm{G}$ network development.

In order to provide a framework for $5 G$ networks, the special issue starts with the paper entitled "A Survey on 5G: The Next Generation of Mobile Communication" [1]. This paper analyses the limitations of current networks in relation to the requirements of $5 G$ and presents a survey of the different key technologies and architectures for $5 \mathrm{G}$.

The next paper, entitled "What will Interference be Like in 5G HetNets?" [2], discusses how the highly dense small cell architectures envisaged for $5 G$ will impact the existing interference patterns. It presents different case studies showing that many of the models existing nowadays can result in potentially highly 
inaccurate predictions of interference and performance when the characteristics of $5 \mathrm{G}$ heterogeneous scenarios are taken into account.

The third paper, entitled "SDN/NFV-enabled Satellite Communications Networks: Opportunities, Scenarios and Challenges" [3], focuses on the integration of the satellite and the terrestrial components in the framework of $5 \mathrm{G}$ networks. It provides a description of the benefits that SDN and NFV technologies can bring into the satellite communications towards $5 \mathrm{G}$, including the identification of relevant scenarios, use cases and associated technical challenges.

The fourth and fifth papers focus on specific radio resource management solutions for different problems of $5 \mathrm{G}$ networks. In particular, the fourth paper, entitled "Inter-Cellular Scheduler for $5 \mathrm{G}$ Wireless Networks" [4], presents a new scheduler for efficient support of multimedia services in multiuser $5 \mathrm{G}$ wireless networks. It dynamically allocates the available bandwidth between the cells to ensure the desired QoS. It also proposes the use of a delay-related metric to measure the resource requirements of each cell. The fifth paper, entitled "Radio Access Technology Selection in Heterogeneous Networks" [5], addresses the problem of selecting the appropriate access technology in a heterogeneous $5 G$ network. It formulates a centralized approach for the decision-making and selection between two broadband technologies and then discusses two distributed approaches, one of them based on heuristics and the other based on a non-cooperative game with a learning-based algorithm. It shows that the distributed algorithms give efficient results compared to the centralized optimal approach.

We hope that the readers will enjoy the papers of this special issue and will use them to gain insight into the different problems and some of the already identified solutions in the area of radio access network architectures and resource management for 5G. We would like to thank all the authors who have submitted papers to this special issue and all reviewers who provided constructive comments to improve the quality of the papers. Finally, we would like to thank the Editor-in-Chief, Prof. Ian Akyildiz, and the Editorial Staff of Elsevier for their support during the preparation of this special issue.

\section{References}

[1] N. Panwar, S. Sharma, A. K. Singh, "A Survey on 5G: The Next Generation of Mobile Communication", Physical Communications, 2015.

[2] J. Riihijärvi, P. Mähönen, M. Petrova, "What will Interference be Like in 5 G HetNets?", Physical Communications, 2015.

[3] R. Ferrús, H. Koumaras, O. Sallent, G. Agapiou, T. Rasheed, M-A. Kourtis, C. Boustie, P. Gélard, T. Ahmed, "SDN/NFV-enabled Satellite Communications Networks: Opportunities, Scenarios and Challenges", Physical Communications, 2015.

[4] C. Gueguen, M. Ezzaouia, M. Yassin, "Inter-Cellular Scheduler for 5G Wireless Networks", Physical Communications, 2015.

[5] K. Khawam, S. Lahoud, M. Ibrahim,M. Yassin, S. Martin, M. El Helou, F. Moety, "Radio Access Technology Selection in Heterogeneous Networks", Physical Communications, 2015. 


\section{Authors' affiliations}

\section{J.Pérez-Romero}

Dept. of Signal Theory and Communications, Universitat Politecnica de Catalunya (UPC), Spain jorperez@tsc.upc.edu

\section{Lagrange}

Dept. Réseaux, Sécurité et Multimédia, Telecom Bretagne / IRISA, France

xavier.lagrange@telecom-bretagne.eu

\section{J. Nasreddine}

Rafik Hariri University, Lebanon

nasreddinejn@rhu.edu.lb

\section{J. Marquez-Barja}

CONNECT -networks of the future- research centre, Trinity College Dublin, Ireland marquejm@tcd.ie

\section{Biographies}

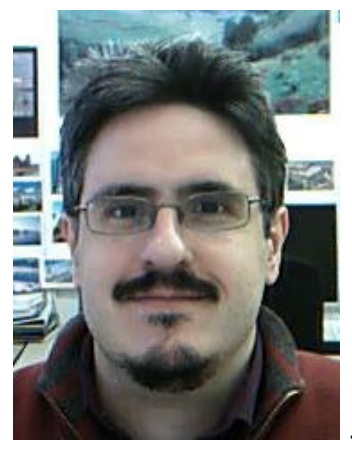

Jordi Pérez-Romero is associate professor at the Dept. of Signal Theory and Communications of the Universitat Politècnica de Catalunya (UPC) in Barcelona, Spain. He received the Telecommunications Engineering degree and the Ph.D. from the same university in 1997 and 2001, respectively. Since then he has been actively working in the field of mobile and wireless communication systems, with particular focus on packet radio techniques, radio resource and QoS management, heterogeneous wireless networks, cognitive radio networks, self-organized networks and network optimization. He has been involved in different European Projects such as EVEREST, E2R, NEWCOM, 
AROMA, E3, FARAMIR, OneFIT, SESAME with different responsibilities as researcher, WP leader and UPC project responsible. He has also participated in different projects for private companies. He has published more than 200 papers in international journals and conferences and has co-authored three books and contributed to 7 book chapters. He holds two international patents and has contributed to 3GPP and ETSI standardization bodies. He is associate editor of two international journals (IEEE Vehicular Technology Magazine and EURASIP Journal on Wireless Communications Networks) and has been TPC in different international conferences. Besides, he participated in the organization of IEEE VTC in Spring 2009 and IEEE PIMRC 2004 international conferences, hold in Barcelona. He has also been TPC Co-chair of EuCNC conference 2014 and of VTC Spring 2015. Moreover, he has organized several workshops special sessions and special issues in international conferences and journals.

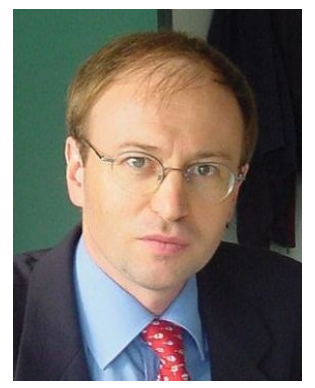

Xavier Lagrange is professor at the departement of Networks, Security and Multimedia in Télécom Bretagne, France. He received the Engineering Degree from Ecole Centrale des Arts et Manufactures, Paris, France, in 1984 and the Ph.D. degree from TELECOM Paristech in 1998. He is the head of research group "Advanced Technologies for Operated Networks" at Institut de recherche en informatique et systèmes aléatoires (IRISA), Rennes, France. His domain of interest includes resource allocation, medium access control, mobility management, performance analysis and new architectures (Cloud Radio Access Networks, convergence between fixed and mobile accesses) for 5th generation networks. He was involved in several national and international cooperative projects (SYSTUF, COMBO). He has published more than 130 papers in international journals and conferences. He has written several books on cellular networks and created several Massive Open Online Courses on the french national portal France Université Numérique. He was TPC co-chair of WPMC 2011.

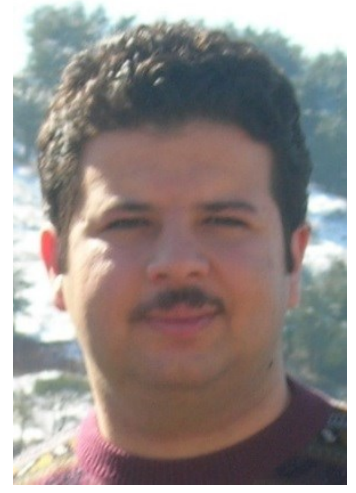

Jad Nasreddine is an expert in radio communication with over 10 years of experience in telecommunication industry and academy. He is presently an Associated Professor at Rafik Hariri University, Lebanon. Previously, he was the RAN/Core product manager at Mobinets, Lebanon. Between 2005 and 2012, he was working as researcher and senior researcher at TelecomBretagne, BarcelonaTech, and RWTH Aachen. He managed and participated in national and industrial projects in France, Spain, and Germany. He also managed and participated in several European projects, international standardization bodies, and international conferences and workshops. He received his Ph.D. degree in March 2005 from university of Rennes I and his B.E. in computer science 
and telecommunications in 2001 from the Lebanese university. During his Ph.D., he was working as research assistant at the Telecom-Bretagne. His research focuses on wireless networks and specifically cognitive radio, self-organizing networks, HetNet, radio environment maps, Fault management, propagation models, and femtocells. He is author of over 50 technical papers in international conferences and journals. He also served as co-chair, associated editor, TPC, and reviewer for several conferences and journals.

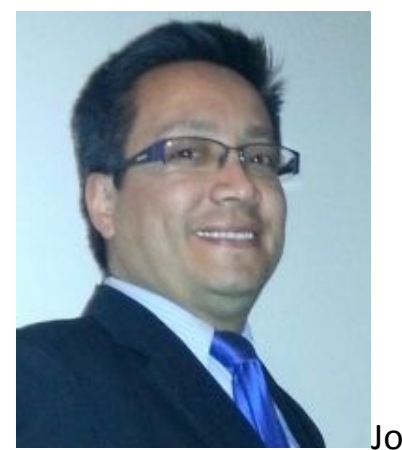
research and education domains. In fact, he is Technical Coordinator for TCD in the FORGE project and Co-Principal Investigator for TCD in the Fed4FIRE/FAVORITE, eWINE, and FUTEBOL projects. He is a member of the IEEE Communications Society as well as the IEEE Education Society, where he participates in the Standards Committee and contributes to the IEEE P1876 - Networked Smart Learning Objects for Online Laboratories standards working group. Dr. Marquez-Barja currently is a Research Fellow at the CONNECT (formerly CTVR) Telecommunications Research Centre at Trinity College Dublin (Ireland) and an adjunct lecturer. His main research areas of interest are advanced heterogeneous wireless/small cells architectures, loT clustering, virtualization, provisioning and dynamic resource allocation. Also, he is interested on vehicular networks, vertical handover techniques and smart cities. Dr. Marquez-Barja is serving as Editor and Guest editor for three International Journals, as well as being part of several Technical Programme and Organizing Committees for different worldwide conferences/congresses. At Trinity College Dublin he lectures on wireless networks and communications systems applying technology-enhanced learning methodologies as part of his educational mission. Dr. Marquez-Barja is an IEEE Senior member of the Communications and Education societies. 